\title{
A Framework for Modeling "Real-Life" Airline Networks
}

\author{
VOLODYMYR BILOTKACH * \\ Department of Economics, University of California, Irvine
}

\begin{abstract}
This paper develops a new model, which allows for the co-existence of hub-and-spoke and point-topoint networks in the airline industry. Passengers are segmented between the non-stop and one-stop services. In the baseline model, the monopoly airline's ability to segment the market and effectively set up the mixed network depends on the cost savings due to the through-hub service relative to exogenous quality difference between the one-stop and non-stop flights. Socially inefficient entry with the new non-stop service where an incumbent is operating the hub-and-spoke network is possible.
\end{abstract}

\section{Introduction}

Literature discussing one of the most dramatic effects of airline deregulation development of hub-and-spoke networks - appears to overlook the fact that, other things equal, a one-stop service is a lower quality product, from the consumer's point of view, than a non-stop flight. More broadly, the issue of network choice by airline(s) has up to now been presented as an "either or" problem of a carrier choosing to either establish a hub or operate a point-to-point network; whereas in "real life" airlines (even those operating hub-and-spoke networks) do choose to provide non-stop services between cities which are not their hubs. This paper offers a general framework for modeling such "real life" airline networks.

Experts started questioning hub-and-spoke networks as a necessary condition for an airline's success on the deregulated market following the rapid expansion of Southwest Airlines, which in addition to being a low cost carrier chose not to rely on a hub-and-spoke network. Several papers (for example, Morrison, 2001; Boguslaski et al., 2004) are devoted to studying this particular airline. Even though the carrier effectively uses several airports as hubs (for example, Phoenix, Chicago Midway, Baltimore), point-to-point services are a more important part of Southwest Airlines' business model than connecting flights. When searching for an explanation of this phenomenon, Berry et al. (1996) turned to the cost side of the problem. Namely, they suggested that Southwest enters routes where economies of traffic density are small or non-existent, so that competitors operating huband-spoke networks will not get any cost advantages over this carrier.

\footnotetext{
* Department of Economics, University of California, Irvine, 3151 Social Science Plaza, Irvine, CA, 92697. E-mail: vbilotka@uci.edu The author thanks the Editor and an anonymous referee for constructive criticism, which has been instrumental in improving the paper.
} 
On the more global scale, the two major aircraft producers, Boeing and Airbus, develop their strategies based on their perception of development of the airlines' networks on the global market. Boeing believes that passengers will want to travel non-stop to more places and focuses on developing more efficient aircraft capable of carrying a modest number of people (around 200-250) as far as possible. Airbus' new creation, A-380, is a manifestation of the company's belief in further development of hub-and-spoke networks, or rather further increase in demand for air travel without fundamental changes in the structure of the airlines' networks.

This paper provides a framework for analyzing the airlines' incentives for segmenting the market for air travel between the one-stop and non-stop services, in an environment where consumers are differentiated by their willingness to pay for shorter flight. More generally, we look at segmentation of a market between two products of exogenous quality with vertical product differentiation. The segmentation is an endogenous feature of our model: Düdden (2006) also suggested segmentation between non-stop and through-hub services as a possibility, but in his model such segmentation was assumed rather than derived as a part of equilibrium.

In general terms, ours is the first model that rationalizes "real-life" airline networks, in which carriers offer non-stop services outside of their hub-and-spoke network structure. Our analysis differs from the literature on network choice by the airline(s) in the following respects. First and foremost, we do not constrain the airline's choice to operating either a hub-and-spoke or a point-to-point network, as done, for example, in Brueckner and Zhang (2001), Pels et al. (2000), Brueckner (2004), Gillen (2006), and Kawasaki (2008) but allow even the monopoly airline to operate both one-stop and non-stop services. Second, unlike in Hendricks et al. (1995), where a monopolist choosing a network to connect a number of cities selects either a hub-and-spoke or a fully connected (point-to-point) network depending on the level of cost savings produced by the former, we find a region of cost differences allowing the monopolist to operate a mixed network. Third, we find that establishing a hub may not deter entry by a firm offering higher quality non-stop service, which is somewhat unlike findings of Oum et al. (1995) ${ }^{1}$. Kawasaki (2008), while differentiating consumers by their value of time as we do here, still constrains airlines to the either-or problem. We achieve these results by recognizing the quality difference between the non-stop and one-stop flights and introducing vertical product differentiation into the model.

Other studies related to our work are Hendricks et al. (1997, 1999), Hu (2005), and Lin (2009). Hendricks et al. (1997) argue that regional carriers are unable to compete with a network carrier operating a hub-and-spoke network. In their 1999 paper, the same authors examine equilibrium network configurations in the case of two airlines competing for passengers on a market for air travel. They determine that aggressive price competition will lead to a monopolist offering a hub-and-spoke network; while if price competition is not aggressive, various (not necessarily hub-and-spoke) network configurations are possible. Hu (2005) shows that with demand uncertainty two airlines competing over a simple three-city network will both choose either hub-and-spoke or point-to-point networks. Lin (2009) considers the issue of legacy carriers' strategy of setting low-cost subsidiaries as a way to counter entry by low-cost airlines, in the network context.

\footnotetext{
${ }^{1}$ We must note, however, that the entrant in our model is constrained to operating the point-to-point network, whereas Oum, Zhang and Zhang do not impose such a restriction.
} 
In our modeling exercise, we start by formally examining the monopolist's incentive to offer both hub-and-spoke and point-to-point services between two cities (thus effectively segmenting the market by the passengers' willingness to pay for higher quality); as well as the possibility of a new non-stop service by an airline, other than the one offering the through-hub flight (modeling this situation as both a simultaneous and a sequential pricesetting game). Whether or not the market will be segmented depends on the relationship between the difference in cost of proving non-stop versus through-hub service on one hand; and the quality difference between the two on the other. In general terms, we find that for every value of quality difference there is a range of the cost difference parameter, where the monopolist will choose to channel its traffic through the hub airport, while the entrant will select to offer new non-stop service (whether post-entry game involves simultaneous or sequential price choice). This way, the market will be segmented by the incumbent and the entrant.

Among the possible extensions of the baseline model, those which will bring us closer to describing reality involve directly modeling economies of traffic density, choice of both prices and frequency, as well as expanding our model to include networks of arbitrary size (the baseline model considers a simple three-city network). We discuss these extensions in rather general terms, pointing to computational difficulties associated with modeling economies of density and frequency.

The rest of the paper is organized as follows. Section 2 presents the model; Section 3 concludes.

\section{$2 \quad$ Model}

\section{$2.1 \quad$ General}

Consider a simple network, as on Figure 1, with three cities (A, B, and H), one of which (city $\mathrm{H}$ ) can serve as a hub for channeling the AB traffic. A typical "network choice" problem in models of this kind relates to the airline choosing whether to operate the nonstop flight between cities A and B. If such a service is offered, all passengers traveling between the two cities will use it; whereas if the $A B$ non-stop service is absent, the passengers will be forced to make a stop en route in $\mathrm{H}$. Thus, $\mathrm{AB}$ market becomes the central one for the purposes of our analysis.

Further, when cost complementarities are absent (that is, size of the AB traffic does not affect the cost of carrying $\mathrm{AH}$ passenger on the corresponding segment of the network); AB market effectively becomes the only object of analysis. Cost complementarities can arise due to economies of traffic density, a well-documented feature of the cost side of the airline industry (Brueckner and Spiller, 1994; Brueckner et al., 1992; and Caves et al., 1984). With such economies, adding an $A B$ passenger will reduce the cost of carrying an $\mathrm{AH}$ passenger, and vice versa. As we will discuss later, modeling economies of density directly is a computationally intensive exercise; in this section we use a legitimate "shortcut" allowing us to model cost savings due to hub-and-spoke network configuration while analyzing the $\mathrm{AB}$ market independently from $\mathrm{AH}$ and $\mathrm{HB}$ routes.

Specifically, we assume constant return to traffic density (so that the cost of carrying each passenger over a segment of the network does not depend on the total traffic on that segment); this assumption allows us to analyze traffic on each market independently, even 
where connecting passengers share the same aircraft with the non-stop customers. To formalize cost savings due to hub-and-spoke network, we will normalize the per passenger cost of connecting A-H-B flight to zero, and denote the same for the non-stop flight through $c \geq 0$. Effectively, $c$ will measure cost savings of operating the hub-and-spoke network relative to offering the non-stop service on $\mathrm{AB}$ market. Setting $c=0$ is equivalent to assuming that channeling traffic through the hub does not produce any cost savings.

We should also note that without the cost complementarities our model can be easily extended to analyze networks of arbitrary size. Specifically, with $M$ end-points of type $A$ (that is, markets $A_{1}, A_{2}, \ldots A_{M}$ such that travel between them through $H$ is not a feasible option) and $N$ end-points of type $B$; we will simply have to solve our problem $\min \{M, N\}$ times.

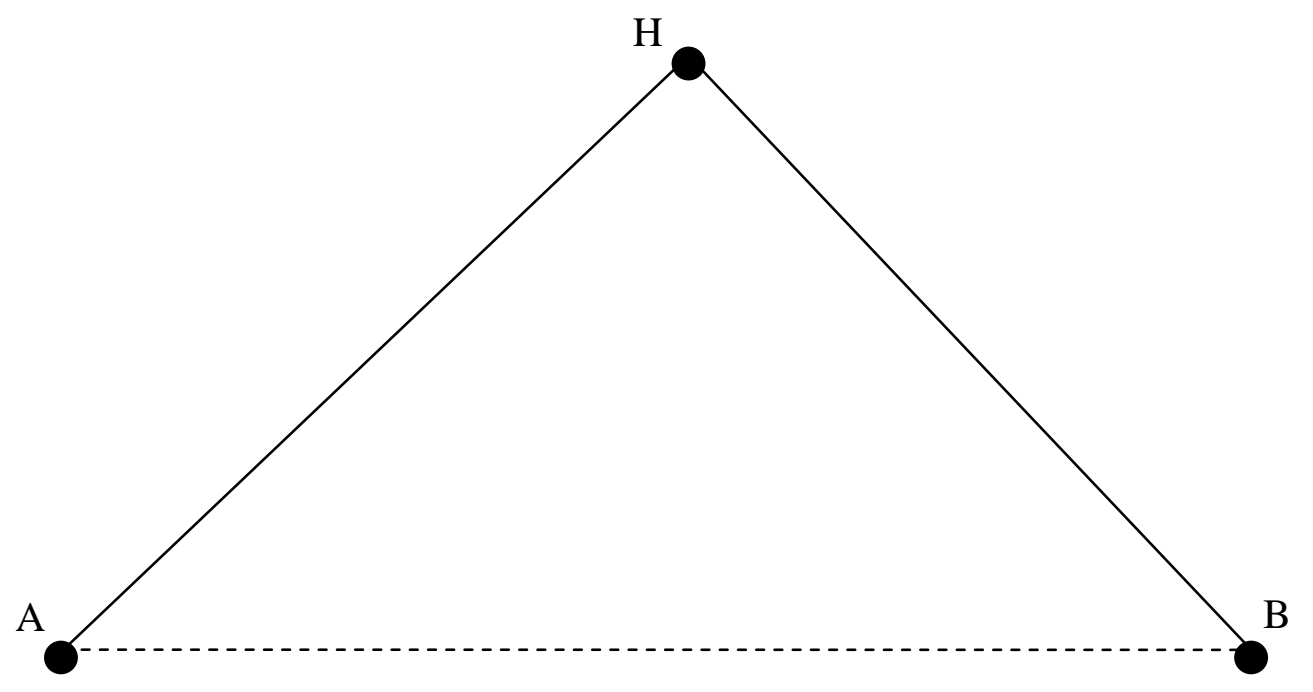

\section{Figure 1: Network}

On the demand side, $\mathrm{AB}$ passengers face two products - trips of different duration. Each consumer makes his choice only once (within some time period), and can either select from the flight options available, or opt for the outside good. Since getting there faster is important for our consumer, he will prefer the non-stop flight to the connecting one, other things equal. Let $t_{H}$ be duration of the flight to/from the hub airport $\mathrm{H}$ (assume A and $\mathrm{B}$ are equidistant from the city $\mathrm{H}$ ), so that total duration of the A-H-B trip is $2 t_{H}$. The non-stop flight is shorter, its duration is denoted through $t_{N S}$ such that: $t_{H}<t_{N S}<2 t_{H}$. Consumers are differentiated by the value of time parameter $\alpha$ in the usual way: $\alpha$ is uniformly distributed on the interval from zero to one. Then, indirect utility of an $\mathrm{AB}$ consumer located at point $\alpha$ is given by

(1) $U_{\alpha}=\left\{\begin{array}{lc}-p_{N S}^{A B}+\alpha\left[T-t_{N S}\right] & \text { if chooses } A B \\ -p_{H}^{A B}+\alpha\left[T-2 t_{H}\right] & \text { if chooses } A H B\end{array}\right.$; 
where $p_{N S}^{A B}$ is the price of the non-stop AB ticket; $p_{H}^{A B}$ is same for the one-stop ticket; and $T \geq 2 t_{H}$ is the total time the passenger can spend at the destination (so that the longer the flight, the less time our consumer can spend doing business or sitting on the beach). Thus, consumer's difference in valuing the two services is affected by the difference in the ticket prices and the passenger's willingness to pay for higher quality, represented by the extra time he will get if he chooses to take the non-stop flight. If we now normalize the value of the most attractive outside option (for example, not travelling or driving instead of flying) to zero, as well as set $T$ to equal one (note that in this case $t_{H} \leq \frac{1}{2}$ ), we can derive demands for non-stop and one-stop travel on the AB market. Generally, consumers with the lowest value of time will choose the outside option; passengers with moderately high $\alpha$ will choose one-stop flight, while those with the highest value of time will travel non-stop. More specifically, location of the consumer indifferent between the outside option and the one-stop flight will be given by

(2) $\hat{\hat{\alpha}}=\frac{p_{H}^{A B}}{1-2 t_{H}}$

while the passenger indifferent between the non-stop and the one-stop flight will be located at:

(3) $\hat{\alpha}=\min \left\{1, \frac{p_{N S}^{A B}-p_{H}^{A B}}{2 t_{H}-t_{N S}}\right\}$.

Given the distributional assumptions on the parameter $\alpha$ introduced above, we obtain the following expressions for demand for one-stop and non-stop services:

(4) $q_{H}=\int_{\hat{\hat{\alpha}}}^{\hat{\alpha}} d \alpha=\min \left\{1, \frac{p_{N S}^{A B}-p_{H}^{A B}}{2 t_{H}-t_{N S}}\right\}-\frac{p_{H}^{A B}}{1-2 t_{H}}$,

(5) $q_{N S}=\int_{\hat{\alpha}}^{1} d \alpha=\max \left\{0,\left(1-\frac{p_{N S}^{A B}-p_{H}^{A B}}{2 t_{H}-t_{N S}}\right)\right\}$.

To address the issue of how the tradeoff between lower cost and lower quality can influence network choice, we will formally consider and compare the following scenarios. First, the case of the monopoly airline will be discussed. The decision to be made by the monopolist is in this case whether or not to segment the market between the non-stop and one-stop services, so that passengers with the higher willingness to pay for quality will use the higher-quality service. Second, we will examine the issue of feasibility of entry with a non-stop service into a market already served by a hub-and-spoke carrier. Specifically, we will examine two versions of the duopoly game, one involving simultaneous choice of prices, and the other one with the hub-and-spoke carrier acting as the price leader. In those cases, AB market will be segmented by two airlines. We will focus our analysis on whether market segmentation by one or two carriers is more likely, and hence how successfully the profit-maximizing monopolist will be able to use segmentation as an entry 
deterrence device; as well as on how the outcomes in the considered cases will relate to the social planner's decision.

\subsection{Monopoly}

Consider a monopolist operating the "mixed" network, in a sense that AB passengers are divided between the non-stop and one-stop services, according to (4) and (5). Then, given the cost structure introduced above, profit function of the monopolist offering both services on the AB market will be

(6) $\pi_{M}=\left(p_{N S}^{M}-c\right) q_{N S}+p_{H}^{M} q_{H}$;

where subscript and superscript $M$ are used to inform the reader we are talking about the monopoly scenario. Then, assuming the 'interior' solution (in a sense that non-stop traffic is strictly positive), (6) becomes

(7) $\pi_{M}=\left(p_{N S}^{M}-c\right)\left(1-\frac{p_{N S}^{M}-p_{H}^{M}}{2 t_{H}-t_{N S}}\right)+p_{H}^{M}\left(\frac{p_{N S}^{M}-p_{H}^{M}}{2 t_{H}-t_{N S}}-\frac{p_{H}^{M}}{1-2 t_{H}}\right)$.

The monopolist then has to choose prices to maximize the profit function (7).

The profit-maximizing prices (after the usual ritual of finding first-order conditions and solving a system of two linear equations) end up as

$$
\begin{aligned}
& \hat{p}_{H}^{M}=\frac{1}{2}\left(1-2 t_{H}\right) \\
& \hat{p}_{N S}^{M}=\frac{1}{2}\left(1+c-t_{N S}\right)
\end{aligned}
$$

which yield the following expressions for the profit-maximizing quantities:

$$
\begin{aligned}
& \hat{q}_{H}^{M}=\frac{c}{2\left(2 t_{H}-t_{N S}\right)} \\
& \hat{q}_{N S}^{M}=\frac{1}{2}\left[1-\frac{c}{2 t_{H}-t_{N S}}\right] .
\end{aligned}
$$

It is obvious from (9) that the monopolist will end up carrying AB passengers through the hub airport $\mathrm{H}$ as long as establishing the hub-and-spoke network produces cost savings relative to the point-to-point network (that is, as long as $c$ is strictly positive). Next, the market will be segmented between the non-stop and the one-stop services as long as the cost savings produced by the hub are small relative to the loss in quality (in terms of difference in travel times) from switching to the hub-and-spoke system. Formally, nonnegative values of $\hat{q}_{N S}^{M}$ require $2 t_{H}-t_{N S}-c \geq 0$.

Our monopolist will thus offer the lower quality good as long as there are cost savings associated with producing it. This means that hub-and-spoke networks will persist as long as channeling passengers through the hub airport is cheaper than flying them non-stop. On the other hand, new technology bringing the cost of non-stop service closer to that of the 
through-hub flight (reducing $c$ in terms of our model's language) will make emergence of the new non-stop service more likely, other things equal.

\subsection{Incentives to enter with new non-stop service}

This sub-section examines the scenario under which the AB market is segmented between two carriers. We motivate our analysis by supposing initially the $A B$ market is monopolized by a carrier offering hub-and-spoke network; another carrier contemplates launching a non-stop service to compete with the incumbent. The case of two airlines competing through hub services (as, for instance, is considered by Oum et al., 1995) is ruled out in the simple case we model, since price competition with homogenous products will lead to the marginal cost pricing and zero profit.

This situation can obviously be modeled in various ways. Setting aside the entry deterrence and predation consideration, the most general modeling approaches (in our simplistic environment where there is no uncertainty and information asymmetry, and long term considerations are omitted) will be the one of incumbent anticipating entry and that of "full" entry accommodation ${ }^{2}$. In the former case, we will model the game as that of sequential choice, with hub-and-spoke firm choosing the price first, and the airline offering non-stop service taking the leader's price as given to make its decision. The latter case will be modeled as the simultaneous choice price competition game with differentiated products.

In either case, the focus of this exercise is as follows. In the previous sub-section, we have established the condition for the monopoly airline to segment the AB market between the non-stop and through-hub services. Here, we will examine if the market segmentation between two carriers is "easier" or "harder" as compared to the monopoly case. The former will be the case if there is a range of cost difference parameter $c$ leading to the entrant opening non-stop service; whereas the monopolist would choose to channel all AB traffic through the hub.

First, consider the sequential price-setting setup. Suppose firm 1 enters first with the A-H-B service (operating hub-and-spoke network), while firm 2 decides whether to enter with the non-stop A-B flight at the second stage of the game. The profit functions are (given that quantities are determined by (4) and (5))

$$
\begin{aligned}
& \pi_{1}=p_{A B}^{H} q_{H}=p_{A B}^{H}\left[\min \left\{1, \frac{p_{A B}^{N S}-p_{A B}^{H}}{2 t_{H}-t_{N S}}\right\}-\frac{p_{A B}^{H}}{1-2 t_{H}}\right] \\
& \pi_{2}=\left(p_{A B}^{N S}-c\right) q_{N S}=\max \left\{0,\left(1-\frac{p_{A B}^{N S}-p_{A B}^{H}}{2 t_{H}-t_{N S}}\right)\right\}\left(p_{A B}^{N S}-c\right)
\end{aligned}
$$

The game is solved by backward induction (first firm 2's profit maximization problem is solved taking firm 1's price as given; and then the solution is substituted into firm 1's profit function, and the profit-maximizing price for firm 1 is determined in the usual way). Again, focusing on the 'interior' solution (so that both services are offered), firm 2's bestresponse function at the second stage of the game will be

\footnotetext{
${ }^{2}$ Economists do disagree which of these general approaches is the "right" one (see, for instance, Martin, 2002, chapter 9) for a discussion.
} 
(11) $\hat{p}_{N S}^{S}=\frac{1}{2}\left(c+p_{H}^{S}+2 t_{H}-t_{N S}\right)$.

This yields the following expression for the profit of firm 1 (the airline offering the through-hub service)

$$
\pi_{1}\left(\hat{p}_{N S}^{S}\right)=p_{H}^{S}\left[\frac{1}{2}\left(1-\frac{p_{H}^{S}-c}{2 t_{H}-t_{N S}}\right)-\frac{p_{H}^{S}}{1-2 t_{H}}\right]
$$

This in turn solves (relatively) easily to yield the following equilibrium prices:

$$
\begin{aligned}
& \hat{p}_{N S}^{S}=\frac{\left(3+2 t_{H}-4 t_{N S}\right)\left(c+2 t_{H}-t_{N S}\right)}{4\left(1+2 t_{H}-2 t_{N S}\right)} \\
& \hat{p}_{H}^{S}=\frac{\left(1-2 t_{H}\right)\left(c+2 t_{H}-t_{N S}\right)}{2\left(1+2 t_{H}-2 t_{N S}\right)},
\end{aligned},
$$

and the corresponding traffic levels will be

$$
\begin{aligned}
& \hat{q}_{N S}^{S}=\frac{1}{4}\left(2+\frac{1-2\left(c+t_{H}\right)}{1-2\left(t_{N S}-t_{H}\right)}-\frac{c}{2 t_{H}-t_{N S}}\right) . \\
& \hat{q}_{H}^{S}=\frac{1}{4}+\frac{c}{4\left(2 t_{H}-t_{N S}\right)}
\end{aligned}
$$

It is intuitive that (as compared to the monopoly case) the game we just solved will yield higher quantity on both the non-stop and the through-hub route (yet, the algebra involved in showing this is somewhat cumbersome).

If providers of non-stop and through-hub services make their choices simultaneously, their profit functions are still given by (10); however, finding the equilibrium of this game involves solving the two firms' first-order conditions as a system of (linear) equations. The solution is, (using the superscript $B$ for "Bertrand-type" game - note that in this case superscript $S$ used above for sequential setup can also be interpreted to stand for "Stackelberg-type" game) assuming both firms provide non-zero traffic in equilibrium

$$
\begin{aligned}
& \hat{p}_{H}^{B}=\frac{\left(1-2 t_{H}\right)\left(c+2 t_{H}-t_{N S}\right)}{\left(3+2 t_{H}-4 t_{N S}\right)} \\
& \hat{p}_{N S}^{B}=\frac{2\left(1-t_{N S}\right)\left(c+2 t_{H}-t_{N S}\right)}{\left(3+2 t_{H}-4 t_{N S}\right)} \\
& \hat{q}_{N S}^{B}=\frac{1}{6}\left(3+\frac{3-4 c-6 t_{H}}{3+2 t_{H}-4 t_{N S}}-\frac{2 c}{2 t_{H}-t_{N S}}\right) \\
& \hat{q}_{H}^{B}=\frac{\left(1-t_{N S}\right)\left(c+2 t_{H}-t_{N S}\right)}{\left(3+2 t_{H}-4 t_{N S}\right)\left(2 t_{H}-t_{N S}\right)}
\end{aligned}
$$


The following Proposition shows how solutions of the simultaneous and the sequential price setting games compare.

Proposition 1. Comparing equilibrium prices and quantities of the simultaneous and sequential price setting games discussed above, we can state the following.

- $\quad$ Prices of both services are higher (and quantities lower) under the sequential choice setup;

- Other things equal, the airline providing through-hub service (the "incumbent") will obtain larger market share under the simultaneous choice setup than with sequential choice;

- Other things equal, the airline providing non-stop service (the "entrant”) will obtain larger market share (and carry more passengers) under sequential choice setup than with simultaneous choice.

Proof: All the results follow directly from comparison of (13) with (15); and (14) with (16).

Technically, our conclusions outlined above should not be surprising in light of the "second mover advantage" result, established for the sequential price competition models of differentiated product oligopoly. It is more interesting to see how our findings regarding segmentation of the market by two airlines relate to same outcome for the case of monopoly. The following Proposition shows that it is "easier" for the new firm to enter the market with the non-stop service than it is for the monopolist to add same to the menu offered to the customers. It also shows that entry with a new non-stop service on a market already served by the incumbent providing through-hub flights will be easier if our hypothetical incumbent is the price leader.

Proposition 2. For both sequential and simultaneous price competition setups analyzed above, the entrant will choose to enter with the non-stop service for $c<2 t_{H}-t_{N S}$. This means that the entrant will choose to enter with the non-stop service where the monopolist will choose not to segment the market. Further, entry with a new non-stop service on a market already served by the incumbent providing through-hub flights will be easier if our hypothetical incumbent is the price leader.

Proof. We established that the monopolist will offer both services if $2 t_{H}-t_{N S}-c \geq 0$. Substituting $c=2 t_{H}-t_{N S}$ into the expressions for non-stop traffic, implied by (14) and (16), we see that

(17) $\hat{q}_{N S}^{S}\left(c=2 t_{H}-t_{N S}\right)=\frac{1-2 t_{H}}{2-4\left(t_{N S}-t_{H}\right)}$;

(18) $\hat{q}_{N S}^{B}\left(c=2 t_{H}-t_{N S}\right)=\frac{1-2 t_{H}}{3-2\left(2 t_{N S}-t_{H}\right)}$. 
Both (17) and (18) are non-negative numbers for $t_{H} \leq \frac{1}{2}$ (so that, since total time available is normalized to one, duration of flights is never larger than duration of the entire trip). This tells us that where the monopolist will choose not to offer the non-stop service, the entrant will select positive non-stop traffic under both setups we considered.

Further, comparing (17) and (18), one will easily conclude that $\hat{q}_{N S}^{S}\left(c=2 t_{H}-t_{N S}\right)>\hat{q}_{N S}^{B}\left(c=2 t_{H}-t_{N S}\right) \quad$ (since $\left.3-2\left(2 t_{N S}-t_{H}\right)>2-4\left(t_{N S}-t_{H}\right)\right)$; this result means that, other things equal, entry with a new non-stop service on a market already served by the incumbent providing through-hub flights will be easier if our hypothetical incumbent is the price leader.

Proposition 2 tells us that there is a range of the differences in the cost parameter $c$, where the profit-maximizing monopolist will not segment the market with both non-stop and one-stop services (offering only the latter); while the entrant (under both the sequential and the simultaneous choice setups) will choose to offer the non-stop service. Furthermore, such range is greater under the sequential choice setup.

\subsection{Social planner}

Next, let us examine the social planner's choice, as well as how it relates to outcomes of the cases considered above. An interesting issue to consider here is the socially optimal market segmentation. It is obvious that the social planner will choose to serve more passengers than under either of the above-discussed scenarios, so that neither the monopolist nor duopoly will offer socially optimal market coverage. However, since conditions for emergence of the new non-stop service are different across the scenarios; we can suspect either (or maybe even both) cases may produce socially inefficient entry.

The social planner will choose (regulated) prices to maximize total market welfare, defined in the usual way as the sum of consumer and producer surplus. The latter will be equal to total profit, since there are no fixed costs. With constant returns to traffic density as we assumed, the social planner will price each of the services at the marginal cost. This in turn means that (using superscript $P$ for "planner")

$$
\begin{array}{ll}
\hat{p}_{H}^{P}=0 ; & \hat{q}_{H}^{P}=\frac{c}{2 t_{H}-t_{N S}} \\
\hat{p}_{N S}^{P}=c ; & \hat{q}_{N S}^{P}=1-\frac{c}{2 t_{H}-t_{N S}}
\end{array}
$$

The social planner will thus serve the entire market (technically, normalizing per passenger cost of through-hub service to zero helped us obtain this result, since the person located at the lower end of the [0,1] interval obtains zero indirect utility). Comparing (19) to (9), one will notice that social planner's choice of output is exactly twice that of the monopolist. Thus, the monopolist will segment the market in such a way that the ratio of non-stop to through-hub passengers will be the same as that chosen by the social planner. Moreover, in deciding whether to segment the market or to offer a single product, both the monopolist and the social planner will use the same criterion. That is, through-hub service will not be offered only if $c=0$; and all passengers will be channeled through the hub airport if $2 t_{H}-t_{N S}-c<0$. 
It is interesting to note that this result will hold for any distribution of the variable $\alpha$, for the linear specification of indirect utility as in (1). The following Proposition shows this formally.

Proposition 3. If consumers' indirect utility function is given by (1), the monopolist will choose the same criterion as the social planner when deciding whether to segment the market between the non-stop and the through-hub services, regardless of the distribution of $\alpha$.

Proof. We have shown above that this result holds for the uniform distribution. Note that the expressions for location of the marginal consumers in the willingness to pay for quality space are invariant to the distribution of $\alpha$ and given by (2) and (3). The demands, however, will be (assuming that both services are offered in equilibrium)

$$
\begin{aligned}
& q_{N S}=1-F(\hat{\alpha}) \\
& q_{H}=F(\hat{\alpha})-F(\hat{\hat{\alpha}})
\end{aligned} ;
$$

where $F$ is the cumulative distribution function (cdf) of the variable $\alpha$. The probability density function will be denoted through the lower-case $f$.

Given this, monopolist's first-order condition with respect to price of the non-stop flight will be

$$
\begin{aligned}
\frac{\partial \pi_{M}}{\partial p_{N S}} & =1-F(\hat{\alpha})-f(\hat{\alpha}) \frac{\partial \hat{\alpha}}{\partial p_{N S}}\left[p_{N S}-c\right]+p_{H} f(\hat{\alpha}) \frac{\partial \hat{\alpha}}{\partial p_{N S}} \\
& =1-F(\hat{\alpha})-f(\hat{\alpha}) \frac{\partial \hat{\alpha}}{\partial p_{N S}}\left[p_{N S}-p_{H}-c\right]=0
\end{aligned}
$$

Then, taking into account (2), (3), (20), and the fact that the social planner will set $\hat{p}_{N S}^{P}=c,(21)$ can be rewritten as

$$
\begin{aligned}
\hat{q}_{N S}^{M} & =f\left(\hat{\alpha}_{M}\right) \frac{\partial \hat{\alpha}}{\partial p_{N S}}\left[p_{N S}-p_{H}-c\right]=f\left(\hat{\alpha}_{M}\right)\left[\frac{p_{N S}-p_{H}-c}{2 t_{H}-t_{N S}}\right] \\
& =f\left(\hat{\alpha}_{M}\right)\left(\hat{\alpha}_{M}-\hat{\alpha}_{P}\right)
\end{aligned}
$$

where $\hat{\alpha}_{M}$ and $\hat{\alpha}_{P}$ are locations of the marginal consumer indifferent between non-stop and one-stop services at the monopolist's and the social planner's optimal choices, respectively. Expression (22) implies that the monopolist will only choose zero non-stop traffic when $\hat{\alpha}_{M}=\hat{\alpha}_{P}$. This is only possible when both are equal to one, as in the other case $^{3}$ we will have $\hat{\alpha}_{M}>\hat{\alpha}_{P}$. Thus, since we cannot have $\hat{\alpha}_{M}<\hat{\alpha}_{P}$; if the social planner chooses not to provide any non-stop service, so will the monopolist.

\footnotetext{
${ }^{3}$ The numerator of alpha is the difference between the non-stop and the one-stop prices. This difference will be equal to the difference in the respective per passenger costs for the social planner's solution and is larger than the cost difference in the two-product monopoly case.
} 
The above result, coupled with Proposition 2, tells us that the oligopoly will produce both socially undesirable entry with the non-stop service, and not socially optimal ratio of non-stop to one-stop passengers. As for the latter, it will be either too high (when the cost difference is large relative to the time difference, so that the planner will choose not to segment the market, while entry will occur), or too low (when the cost difference approaches zero). In the extreme case of no per passenger cost difference between one-stop and non-stop flights, duopoly produces positive one-stop traffic (see (14) and (16)), whereas the social planner (as well as the monopolist - see (9)) will choose not to segment the market and carry all the passengers non-stop.

\subsection{Extensions}

\subsubsection{Economies of density}

Of the aspects in which our modeling exercise inevitably differs from the naturally occurring situation, the most problematic one is lack of direct modeling of such widely recognized benefits of establishing the hub-and-spoke network as better exploitation of economies of traffic density and ability to offer higher frequency of service.

With economies of traffic density (a feature of the airline industry well documented empirically by Brueckner and Spiller (1994), Brueckner et al. (1992), Caves et al. (1984), and Berry et. al (1996)), per passenger cost on each segment of the hub-and-spoke network decreases with the increase in number of through-hub passengers. Thus, with economies of density incorporated into our setup, we would obtain a truly network model. An almost canonical approach in the literature involves assuming marginal cost function of the form

(23) $M C(Q)=1-\theta Q$,

where $\theta$ is the measure of extent of economies of traffic density (the larger this number, the more an extra passenger adds to decline in marginal cost). Notice that marginal cost is defined for segment rather than market level, and $Q$ refers to total traffic on the segment. In the context of our model, we would define (23) for both $A H$ and $H B$ segments; and total traffic levels would be: $Q_{A H}=q^{A H}+q_{H}^{A B}$ and $Q_{H B}=q^{H B}+q_{H}^{A B}$. When this marginal cost function is introduced, closed-form solutions can be found; however, these will be so cumbersome they will not allow for any meaningful comparative statics comparisons.

Previous modeling exercises (for example, Brueckner and Spiller, 1991 and Bilotkach, 2007) resorted to numerical simulations to trace comparative statics in models where economies of density play a crucial role. We can use lessons learned in those studies to claim that outcome of analysis directly modeling economies of density within our framework will depend on the extent of density parameter; the larger the $\theta$, the more difficult it will be for the monopolist to segment the market; and the more difficult the entry with the non-stop service will be, other things equal. Qualitatively, however, introducing economies of density will not introduce new results into our model, beyond, possibly, welfare implications (which will again depend on $\theta$, as they have in the above cited models). 


\subsubsection{Including frequency of service}

We started this paper by saying that one-stop service is a lower quality product as compared to non-stop one, other things equal. In the real world, however, operating a huband-spoke network allows the airline to increase frequency of its services (which is an important determinant of quality from the consumers' point of view, since higher frequency increases the chance of departing closer to one's preferred time), so that if we allow the carrier(s) to choose both price and frequency, the one-stop service may not end up being a lower quality product after all. Here we would again face the intractability trap, however. In general, models of vertical product differentiation not putting restrictions on the quality dimension require either convex cost or decreasing marginal utility of quality, to prevent firms from choosing infinite quality in equilibrium ${ }^{4}$. Then, any asymmetries between the firms complicate the problem substantially, making it impossible to obtain a closed-form solution.

The two frameworks for modeling competition in prices and frequency have been proposed recently by Heimer and Shy (2006) and Brueckner and Flores-Fillol (2007). The former uses linear marginal utility and convex cost of frequency; while the latter assumes decreasing marginal utility of frequency with constant per flight cost. Both models offer tractable solutions only for symmetric equilbria (that is, where both airlines choose the same flight frequency). Flores-Fillol (2009) uses a similar framework to Brueckner and Flores-Fillol's to analyze the issue of competing airlines' choice of network structure (while still not allowing the carriers to choose mixed networks of the kind discussed here). Bilotkach et al. (2008) supplement Brueckner and Flores-Fillol's model with consumer heterogeneity of the kind introduced in this study to examine the choice of a monopoly provider of scheduled transportation services (for example, airline company or high-speed rail operator) facing consumers who have an option of an alternative entailing lower speed but higher level of mobility (that is, personal vehicle).

The above-mentioned models have proven to be analytically intractable when solutions are asymmetric; inserting frequency into our framework encounters an additional obstacle - dependence of equilibrium frequency on demand on AH and HB markets. There is also an issue of correct specification of the frequency of through-hub service on $A B$ market as a function of $\mathrm{AH}$ and $\mathrm{HB}$ frequencies (bottleneck approach, whereby $\mathrm{AB}$ frequency is the minimum of the two appears the most appealing).

\section{$3 \quad$ Concluding comments}

This paper offers a new look at the issue of airlines' choice of network structure. Unlike the previous literature modeling choice between hub-and-spoke and point-to-point network, we allow for segmentation of the market between non-stop and one-stop services. Basically, we acknowledge the fact that, other things equal, a one-stop flight is a lower quality product than a non-stop service. We then use a simple model of vertical product differentiation to develop a framework for modeling networks in which there is market segmentation between a non-stop and a one-stop flight. Such networks are more

\footnotetext{
${ }^{4}$ The textbook model with constrained quality space leads to the maximum product differentiation equilibrium with constant marginal utility of quality, even if quality is costless (for example, Shy, 1996).
} 
representative of the airlines' actual choices than purely hub-and-spoke or purely point-topoint setups.

The findings of our analysis are the following. First, where both through-hub and nonstop services are feasible, there is a possibility for segmenting the market so that the customers with higher willingness to pay for quality will choose the non-stop flight, and those with lower willingness to pay will fly through the hub airport. Second, whether the segmentation will take place depends on the cost savings associated with the one-stop service relative to the quality difference between one-stop and non-stop flights. Third, the monopolist deciding whether to segment the market and effectively operate the mixed network will use the same criterion as the social planner. Fourth, other things equal, cost advantages a hub-and-spoke incumbent might have over the non-stop entrant may not deter such an entry; keeping the quality difference constant, there are values of difference in cost parameter, which are too high for the incumbent to consider adding non-stop service, but sufficiently low for the entrant to offer same.

More realistic but unfortunately intractable setups would involve modeling of economies of traffic density (incorporating this aspect of airlines' cost structure directly one will both offer a truly network setup in which through-hub and direct passengers are taken into account, and can potentially address such issues as factors influencing airlines' choice of hub airports) and/or frequency choice. We provide some discussion of these aspects of the problem, reviewing approaches suggested in the literature, as well as problems encountered in previous studies.

This study establishes a framework, and it is clear that there is a lot of room for improving on our setup. Notably, the extensions we touch on here are tedious to analyze; yet, they can potentially yield interesting results. This is the broader issue we leave for the future research endeavors.

\section{$4 \quad$ References}

Berry, S., M. Carnall and P. Spiller (1996) “Airline Hubs: Costs, Markups, and the Implications of Customer Heterogeneity,” NBER Working Paper 5561.

Bilotkach, V. (2007) "Complementary versus Semi-Complementary Airline Alliances," Transportation Research B, 41: 381-393.

Bilotkach, V., X. Fageda, and R. Flores-Fillol (2008) "Scheduled Service versus Private Transportation: the Role of Distance,” working paper, University of Barcelona.

Boguslaski, C., H. Ito and D. Lee (2004) "Entry Patterns in the Southwest Airlines Route System,” Review of Industrial Organization, 25: 315-340.

Brueckner, J. (2004) “Network Structure and Airline Scheduling,” Journal of Industrial Economics, 52: 291-312.

Brueckner, J., N. Dyer, and P. Spiller (1992) "Fare Determination in Airline Hub-andSpoke Networks,” RAND Journal of Economics, 23: 309-333. 
Brueckner, J., and R. Flores-Fillol (2007) "Airline Schedule Competition: Product-Quality Choice in a Duopoly Model,” Review of Industrial Organization, 30: 161-177.

Brueckner, J., and P. Spiller (1991) "Competition and Mergers in Airline Networks," International Journal of Industrial Organization, 9: 323-342.

Brueckner, J., and P. Spiller (1994) "Economies of Traffic Density in the Deregulated Airline Industry,” Journal of Law and Economics, 37: 379-415.

Brueckner, J., and A. Zhang (2001) "A Model of Scheduling in Airline Networks: How a Hub-and-Spoke System Affects Flight Frequency, Fares, and Welfare," Journal of Transport Economics and Policy, 35: 195-222.

Caves, D., L. Christensen, and M. Tretheway (1984) "Economies of Density versus Economies of Scale: Why Trunk and Local Service Airline Costs Differ," RAND Journal of Economics, 15: 471-489.

Düdden, J-C. (2006) "Multi-Hub Network Configurations - A Temporary or Permanent Outcome of Airline Consolidation?” Review of Network Economics, 5: 421-434.

Dunn, A. (2007) "Do Low-Quality Products Attract High-Quality Entry: Multiproduct Firms and Non-Stop Entry in Airline Markets," International Journal of Industrial Organization, 26: 1074-1089.

Flores-Fillol, R. (2009) “Airline Competition and Network Structure,” Transportation Research $B$, forthcoming.

Heimer, O., and O. Shy (2006) "Code-Sharing Agreements, Frequency of Flights, and Profits under Parallel Operation," in Darin Lee (ed.) Advances in Airline Economics, Elsevier: Amsterdam.

Hendricks, K., M. Piccione, and G. Tan (1995) "The Economics of Hubs: The Case of Monopoly,” Review of Economic Studies, 62: 83-99.

Hendricks, K., M. Piccione, and G. Tan (1997) "Entry and Exit in Hub-and-Spoke Networks,” RAND Journal of Economics, 28: 291-303.

Hendricks, K., M. Piccione, and G. Tan (1999) “Equilibria in Networks,” Econometrica, 67: 1407-34.

Hu, Q. (2005) “Are Hub-and-Spoke Networks Better Than Point-to-Point Networks?” Case Western Reserve University Department of Operations Technical Memorandum Number 800.

Gillen, D. (2006) “Airline Business Models and Networks: Regulation, Competition, and Evolution in Aviation Markets,” Review of Network Economics, 5: 366-385.

Kawasaki, A. (2008) "Network Effects, Heterogeneous Time Value, and Network Formation in the Airline Market," Regional Science and Urban Economics, 38: 388-403. 
Lin, M-H. (2009) “Airlines-within-Airlines Strategies and Entry of Low Cost Airlines,” working paper, Chukyo University.

Martin, S. (2002) Advanced Industrial Economics, $2^{\text {nd }}$ Edition. Blackwell: London.

Morrison, S. (2001) “Actual, Adjacent, and Potential Competition: Estimating the Full Effect of Southwest Airlines,” Journal of Transport Economics and Policy, 35: 239-256.

Oum, T., A. Zhang, and Y. Zhang (1995) “Airline Network Rivalry,” Canadian Journal of Economics, 28: 836-857.

Pels, E., P. Nijkamp and P. Rietveld (2000) "A Note on the Optimality of Airline Networks," Economics Letters, 69: 429-434.

Shy, O. (1996) Industrial Organization: Theory and Applications. The MIT Press: Cambridge, MA. 